\title{
GESTIÓN DE UNA COMUNIDAD DE PRÁCTICA. CASO DE ESTUDIO: INCIDENTES DE INFRAESTRUCTURA DE DOS PYME DE LA CIUDAD DE MEDELLÍN
}

\author{
Managing a Community of Practice. Case study: infrastructure incidents at two SMEs in \\ Medellín, Colombia
}

Fabián Mauricio Vélez-Salazar

Magíster en Gestión Tecnológica. Instituto Tecnológico Metropolitano. Medellín-Colombia, mauriciovelez@itm.edu.co

\begin{abstract}
Gustavo Adolfo Duque-Osorio
Magíster en Gestión de la Innovación Tecnológica, Cooperación y Desarrollo Regional. Instituto Tecnológico Metropolitano. Medellín-Colombia, gustavoduqueo@gmail.com
\end{abstract}

\section{Cómo citar / How to cite}

Vélez-Salazar, F. M. y Duque-Osorio, G. A. (2017). Gestión de una comunidad de práctica. Caso de estudio: incidentes de infraestructura de dos pymes de la ciudad de Medellín. Revista CEA, 3(5), 59-68.

Recibido: 25 de julio de 2016

Aceptado: 25 de septiembre de 2016

\section{Resumen}

A diario, las grandes y pequeñas compañías generan gran cantidad de información, datos de toda índole, relevantes e irrelevantes para la misma, desconociendo el enorme potencial que contiene. A través de este trabajo se presentan los resultados de la gestión de la información en una comunidad de práctica (CoP) a partir de un sistema de monitoreo que recibe como insumo la información básica de equipos y los datos de incidentes presentados en los mismos, de dos empresas dedicadas a la importación y comercialización de productos químicos en la zona sur del valle de Aburrá.

Con este trabajo se pretendió compensar la falta de información sobre las características de las tecnologías usadas en ese entorno empresarial, los incidentes y las fallas más comunes, entre otras, por medio de una comunidad de práctica, donde se almacena la información arrojada por el sistema de monitoreo y se gestiona entregando los argumentos necesarios para la administración de las fallas, de su diagnóstico, de planes de mantenimiento preventivo y correctivo, etc., basados en reportes e incidentes reales generados por las compañías que hacen parte de dicha comunidad. Esta información obtenida y gestionada, a su vez será analizada y utilizada para estudios de futuro y toma de decisiones de las empresas.

Palabras clave: comunidades de práctica, sistemas de monitoreo, infraestructura tecnológica, inteligencia de negocios, gestión del conocimiento. 


\section{Abstract}

Big and small companies daily produce large amounts of information, relevant and irrelevant data of all kinds; but they ignore the enormous potential in it. This work presents the results of an information management strategy in a Community of Practice (CoP) that employs a monitoring system whose input is the basic information regarding equipment and incidents at two companies that import and commercialize chemical products in the South of Aburrá Valley.

This work was intended to collect missing information on the characteristics of the technology used in this business environment and the most common incidents and failures, among others. A community of practice stored the information provided by the monitoring system and the data were managed by supplying the necessary criteria for administering failures, diagnoses, preventive and corrective maintenance plans, and so on, based on real reports and incidents by companies in said community. The information that was collected and managed by the community of practice will in turn be analyzed and used for future studies and corporate decision-making.

Keywords: communities of practice, monitoring systems, technological infrastructure, business intelligence, knowledge management.

\section{INTRODUCCIÓN}

Una Comunidad de Práctica (CoP) puede ser definida como «un grupo de personas ligadas por una práctica común, recurrente y estable en el tiempo» (Vásquez, 2011); personas que comparten una preocupación o una pasión por un tema particular y que interactúan de manera continua buscando ahondar en el conocimiento de dicho tema (Cox, 2005). Gracias a este interés común, los miembros de la comunidad aprenden permanentemente del entorno práctico y de la experiencia que los demás comparten en ella. Debido a los avances tecnológicos actuales, existen herramientas que facilitan la interacción entre los miembros de una CoP, como internet y las aplicaciones web. Estos elementos ayudan a superar las barreras de ubicación geográfica y de idioma, entre otras (Vásquez, 2011).

Sin embargo, todas estas herramientas requieren de una importante interacción de los participantes, lo que se traduce en una demanda de tiempo significativa para mantener actualizada la comunidad.

Por lo tanto, una comunidad de práctica se define como un grupo que se cohesiona a través del compromiso mutuo y de la creación de un repertorio común (Cox, 2005).

La responsabilidad de pertenecer a una comunidad de práctica radica esencialmente en la participación activa en la misma, con elementos enriquecedores del conocimiento que puede ser generado dentro del grupo, de ahí la estrecha relación entre las CoP y la gestión del conocimiento. Una comunidad de práctica exitosa tiene una alta interacción entre los miembros que conforman la misma (Vásquez, 2011).

Este trabajo presenta una «automatización» de la información de Infraestructura Tecnológica (IT) -relacionada con el inventario de hardware y software instalado, uso, rendimiento e incidentes presentados y su correspondiente solución, entre muchos otros aspectos, de dos PYME de comercio exterior domiciliadas en la zona sur del valle de Aburrá-, suministrada por el sistema de monitoreo de cada uno de los miembros de la comunidad conformando una base de datos de conocimiento útil para el análisis y la toma de decisiones generando acciones futuras y acertadas en la organización. 
Un mismo problema de IT puede presentarse en varias compañías, y no existe la disposición ni el tiempo necesario para compartir esta información y construir una base de conocimiento que permita mejorar los tiempos de respuesta y la presentación de soluciones oportunas. Las comunidades de práctica permiten centralizar y compartir las mejores prácticas, diagnósticos, datos estadísticos y cualquier otra información de interés común entre sus miembros. De ahí su importancia y valor en la actualidad.

\section{COMUNIDAD DE PRÁCTICA}

En la década de los 80 se ve la aparición de las primeras comunidades de aprendizaje (Preece et al., 2003) que encaminaban los intereses habituales de la comunidad académica en un mismo lugar. La realidad tecnológica de comunicación, como Internet y la mensajería instantánea, facilitaron el crecimiento de este concepto (Droschl 2004) y el reconocimiento al potencial del trabajo colaborativo, así las comunidades de práctica fue tomando forma aprovechando las bondades de la World Wide Web, como el caso del gigante de las comunicaciones, Cisco Systems Inc, quien revolucionó las plataformas de enseñanza y aprendizaje en línea, con sus herramientas basadas en tecnologías web 2.0 (Frezzo et al., 2008).

Las CoP han sido utilizadas en diferentes disciplinas a través del tiempo. En la medicina, por ejemplo, han sido de gran ayuda para el intercambio y la conformación de bases de datos de conocimiento; Ghosh et al. (2009) muestran la aplicación de una comunidad de práctica, con el fin de mejorar la calidad de los datos hospedados en un sistema integrado para el cuidado de la salud; la incorporación de técnicas de inteligencia artificial y sistemas expertos direccionan el camino de las CoP y las demás herramientas de gestión del conocimiento (Berkani et al., 2013); y en general, las Tecnologías de la Información y la Comunicación (TIC) han ingresado en la sociedad en pleno, desde el individuo hasta el contexto empresarial, que cada vez las empresas, sin importar su tamaño, implementan prácticas relacionadas con TIC y obtienen un acceso más eficiente a la información disponible en el medio, realizan una mejor gestión del conocimiento interno y externo, pueden lograr la reducción de costos en sus procesos, monitorear y controlar los negocios de la compañía con mejores resultados y mantienen información actualizada sobre el estado de la corporación y su entorno, mencionando solo algunos de los beneficios (De León, Villa, Vázquez \& Salcedo, 2014).

Sin embargo, no siempre la implementación de herramientas asociadas a las TIC cumple con su objetivo principal. El desconocimiento de las bondades de la gestión de la información no permite que dichas herramientas sean aprovechadas al máximo, y para que la gente acepte entregar y crear conocimiento colectivo deben darse algunas condiciones, como el reconocimiento público a quienes comparten sus conocimientos y un nivel de confianza entre los miembros de la comunidad. Cuando los integrantes de diferentes empresas y disímiles disciplinas realizan proyectos conjuntos y comparten todas sus experiencias, se crea confianza entre ellos, razón por la cual, en las comunidades de práctica, transita de manera fluida el conocimiento, creando escenarios apropiados para la generación compartida del mismo. Una vez establecida la relación de confianza, a través de las comunidades de práctica, se acrecienta el capital social con el establecimiento de redes de personas con intereses comunes (Vásquez, 2011).

De otro lado, en las comunidades de práctica existen unas características importantes que deben ser tenidas en cuenta en el momento de su implementación: su creación debe ser casi 
espontánea: debe existir un deseo o una necesidad de trabajo sobre un tema particular que despierte suficiente interés en los participantes como para seguir aportando conocimiento constantemente en la comunidad creada, inclusive, utilizando recursos diferentes a la misma CoP -como foros virtuales, directorios de especialistas en temas particulares, metodologías de trabajo virtual y presencial- para el intercambio de información, con el objetivo de enriquecer intelectualmente a cada uno de sus participantes; la definición de los temas de trabajo de la CoP: es importante evitar la dispersión de los participantes acerca del tema central de la comunidad, sin embargo, esto no impide que la CoP evolucione y se hallen temas afines con el tópico de creación de la misma; un moderador: un miembro de la comunidad que realice el papel de animador que pueda valorar los aportes de los participantes, que aprecie las contribuciones realizadas en la comunidad y las relaciones creadas alrededor de la misma; los niveles de participación: los apasionados que participan de todos los temas relacionados en la comunidad, los activos que participan en las reuniones presenciales y siguen los aportes en la comunidad pero solo intervienen esporádicamente y los miembros que aparecen como observadores pasivos quienes asimilan los conocimientos y los colocan en práctica (Vásquez, 2011).

De igual forma, pueden existir conversaciones privadas dentro de la comunidad de práctica, fundamentales para generar confianza entre los integrantes de la comunidad al identificar la credibilidad de cada uno de los integrantes en relación a sus pares en la CoP, lo que se convierte en el corazón de la comunidad de práctica, puesto que influye directamente en el desarrollo de la misma. Esta forma de trabajo conjunto es lo que conlleva a replicar en diferentes escenarios la manera de solucionar los inconvenientes tratados en la comunidad (Barragán, 2015).
Sin embargo, teóricamente es muy difícil explicar la razón que impulsa a los integrantes de las comunidades de práctica a promover ese trabajo conjunto, a gestionar la negociación del trabajo y esfuerzo conjunto a su favor, organizar, identificar y promover sus propios intereses en el trabajo e inclusive, en algunos casos, para jugar o compartir claves importantes en juegos comunes (Cox, 2005).

Las razones antes mencionadas resultan de gran interés a las personas que trabajan en gestión del conocimiento -como profesionales o investigadores-, de ahí la relación directa entre las CoP y GC por la dificultad que presentan los proyectos de GC para ser compartidos, situación que es solucionada por las CoP-camino de práctica compartida-al ser estructuras en las cuales el conocimiento fluye, se comparte y circula fácilmente (Vásquez, 2011), es decir, se aplica lo aprendido propiciando un ambiente en el cual surgen aprendizajes útiles y totalmente adaptables en escenarios similares (Barragán, 2015). No obstante, existen problemas de difícil solución -por su dimensión tácita- que no pueden ser planteados, ni compartidos, ni adquiridos por medio de la participación en una CoP (Vásquez, 2011).

En tiempos anteriores, los sistemas informáticos permitieron realizar algunos proyectos de gestión del conocimiento porque ayudaban a capturar, almacenar, buscar y distribuir el conocimiento, sin embargo, la mayoría de estos proyectos naufragó puesto que de un lado no era tan elemental aprehender el conocimiento de las personas, y del otro, cuando el conocimiento era almacenado, este ya no era utilizado por los interesados, asumiendo erróneamente que la generación de conocimiento y la forma de compartirlo eran evidentes, resultando en las dificultades inherentes a las comunidades de práctica como que las personas en muchas ocasiones no quieren compartir sus ideas, 
mejores prácticas, en general su conocimiento, por temor a perder vigencia como empleados; la incompetencia: la gente piensa que son mal percibidos por utilizar ideas de otras personas; el aprieto asociado al tiempo: debe dedicarse un poco de tiempo adicional al laboral para escribir las mejores prácticas y publicarlas en la comunidad de práctica, idea que no es muy bien recibida usualmente; y la asimilación del conocimiento: mezcla la práctica y repetición de actividades conjuntas durante un tiempo para compartir el saber, situación que se presentada satisfactoriamente en las comunidades de práctica (Vásquez, 2011).

Adicionalmente, las Tecnologías de la Información y la Comunicación ayudan en la conformación de las comunidades de práctica (Barragán, 2015) a través de la creación de directorios o repositorios de información, para que por medio de ellas, las personas poseedoras de conocimiento común establezcan las relaciones de confianza necesarias para la generación de conocimiento compartido; de hecho, la Infraestructura Tecnológica (IT) -hardware y software- es uno de los asientos fundamentales en las Tecnologías de la Información y la Comunicación y, como muchos elementos que hacen parte de la organización, debe ser monitoreada y controlada con el fin de medir su rendimiento, diagnosticar posibles fallas, proporcionar los componentes necesarios para elaborar planes de mantenimiento preventivo y correctivo y elaborar informes sobre las mejores y peores prácticas de IT.

Un marco de trabajo colaborativo compromete al usuario a realizar aportes a la comunidad y le permite aprovechar la información generada por otros usuarios. La construcción de una base de datos compartida amplía el campo muestral objeto de cualquier análisis, beneficiando a todos los miembros de dicha comunidad y generando una sinergia con resultados superiores a los que pueden lograrse con información individual. Para el caso de estudio particular, la información generada por varias empresas centralizada en una base de datos brinda un enfoque más amplio que la misma información fraccionada para cada compañía en particular.

Las decisiones que toman los administradores de Infraestructura Tecnológica (IT) de las PYME, normalmente no están soportadas por herramientas precisas que garanticen la información necesaria y suficiente, ya que este tipo de compañías no cuenta con la capacidad económica apta para invertir en este tipo de soluciones. El Project Management Body of Knowledge PMBOK (PMI, 2008), contiene las mejores prácticas relacionadas con la administración de proyectos y cuenta con un proceso exclusivo para la gestión de las adquisiciones, dando especial importancia a la correcta planificación de las mismas. Una correcta gestión de este aspecto garantiza que las inversiones hechas en IT están alineadas con el mapa estratégico de la compañía y la generación de valor futuro.

Las compañías generan diariamente gran cantidad de información de IT relacionada con el inventario de hardware y software instalado, uso, rendimiento e incidentes presentados, con su correspondiente solución, entre muchos otros aspectos. Si bien, un mismo problema de IT puede presentarse en varias compañías, no existe la disposición ni el tiempo para que se comparta esta información y se construya una base de conocimiento que les permita mejorar los tiempos de respuesta y la presentación de soluciones oportunas. En este caso, la construcción de una CoP permite centralizar y compartir las mejores prácticas, diagnósticos, datos estadísticos y cualquier otra información de interés común entre los miembros. De ahí la importancia y valor en la actualidad. 


\section{METOdOLOGÍA}

El proyecto se enfocó en la creación, alimentación y gestión de una comunidad de práctica, en la cual reposan los datos arrojados por los sistemas de monitoreo de las empresas caso de estudio y que hacen parte esencial de la información -posibles fallos, rendimiento, planes de mantenimiento, etc. - que será compartida y convertida en mejores prácticas por los integrantes de la comunidad.

A través de los sistemas de monitoreo instalados en las dos PYME elegidas -los cuales se conectan con la plataforma central de la CoP con el fin de intercambiar la información-, se obtienen los reportes de inventario de hardware y software instalado, uso, rendimiento y la base de conocimiento construida a partir de la gestión de incidentes, presentados y su correspondiente solución de IT. Los flujos de trabajo del proceso fueron el modelado del negocio: para entender las reglas del ejercicio de la empresa y el funcionamiento general del proceso de monitoreo de infraestructura tecnológica de las PYME elegidas buscando la contextualización de las personas encargadas de los procesos de IT; análisis y diseño: arquitectura del sistema, teniendo en cuenta el cumplimiento del modelado del negocio y describiendo la implementación del mismo; requerimientos: exigencias de los usuarios de la comunidad de práctica, es decir, la caracterización de la información que se quiere obtener de los sistemas de monitoreo y cualquier otra información que el usuario final considere relevante como el tipo de dato, origen, periodicidad, temporalidad, etc.; implementación: construcción de los elementos necesarios del sistema de monitoreo y la integración con la comunidad de práctica; pruebas: garantizan la calidad de la solución desarrollada; y despliegue: instalación, adaptación y parametrización del sistema de monitoreo de 2 PYMES y la integración con la comunidad de práctica.

El buen funcionamiento de la compañía depende en gran parte de la infraestructura tecnológica, razón de peso del monitoreo. Los elementos monitoreados más importantes fueron el uso de la CPU, uso de memoria, tráfico de red (recepción y envío), espacio disponible (servidores y sistemas de almacenamiento) y los eventos (suministrados por el sistema operativo). La gestión de incidentes es una actividad que se rige por un estándar internacional ITIL (Information Technology Infrastructure Library). De acuerdo con este estándar, un incidente podría definirse como «cualquier evento que no forma parte de la operación estándar de un servicio y que causa, o puede causar, una interrupción o una reducción de calidad del mismo» (Oficina de Comercio del Gobierno Británico, 2011). Los incidentes tienen un ciclo de vida que se inicia con la identificación del evento, bien sea por información de un usuario que lo detectó o por una alerta generada por un sistema de monitoreo. Una vez se identifica que existe un evento anómalo, se procede a clasificar el incidente. Posteriormente, es necesario determinar la prioridad del incidente, dependiendo del impacto y la urgencia del evento presentado. El impacto puede determinarse dependiendo de la criticidad del servicio afectado y la cantidad de usuarios que están involucrados. La urgencia depende más del tiempo de resolución que el usuario o cliente esté dispuesto a aceptar, y normalmente está consignado en un documento denominado SLA (Service Level Agreement) o Acuerdo de Niveles de Servicio. Debido a que todas las compañías tienen diferentes servicios y su criticidad, varía de acuerdo con el negocio, no existe un estándar para este punto. El incidente tiene una descripción clara del evento que permite al administrador identificar el contexto de la situación que se está presentando. Una vez 
registrado el incidente, se procede con el escalamiento correspondiente.

Como este caso de estudio se desarrolló en PYME y no cuentan con un área compleja de IT, el mismo administrador de IT es quien se encarga de darle gestión al incidente $y$ escalarlo a un proveedor, si es el caso. Los estados tenidos en cuenta fueron: en diagnóstico, solución en progreso, escalado a proveedor, en pruebas, cerrado. Para este caso, uno de los aspectos más importantes es la documentación generada en el proceso de diagnóstico y solución de los incidentes, ya que esta es la materia prima para la CoP. Así, mientras el administrador de IT documenta sus incidentes y construye una base de conocimientos propia, también hace un aporte valioso a los demás miembros de la CoP, sin tener que invertir tiempo extra. Luego, desde los sistemas de monitoreo fue necesario realizar configuración que permitió recolectary almacenar la información que es integrada en la comunidad de práctica para su respectiva publicación.

La Fig. 1 describe el proceso de integración entre los sistemas de monitoreo de las PYME y la comunidad de práctica: la exportación de la información necesaria requerida para la integración desde los sistemas de monitoreo; la transferencia o importación de los datos hacia la comunidad de práctica: inicialmente se realiza un proceso de borrado de los registros de las PYME en la CoP, con el fin de garantizar la integridad de la información y evitar la duplicidad de los datos; posteriormente, se procede con la inserción de los registros correspondientes y por último se eliminan los archivos de origen. Una vez superada la fase de integración, los usuarios podrán autenticarse en la CoP para consultar reportes, incidentes y soluciones de todos los miembros.
(2)
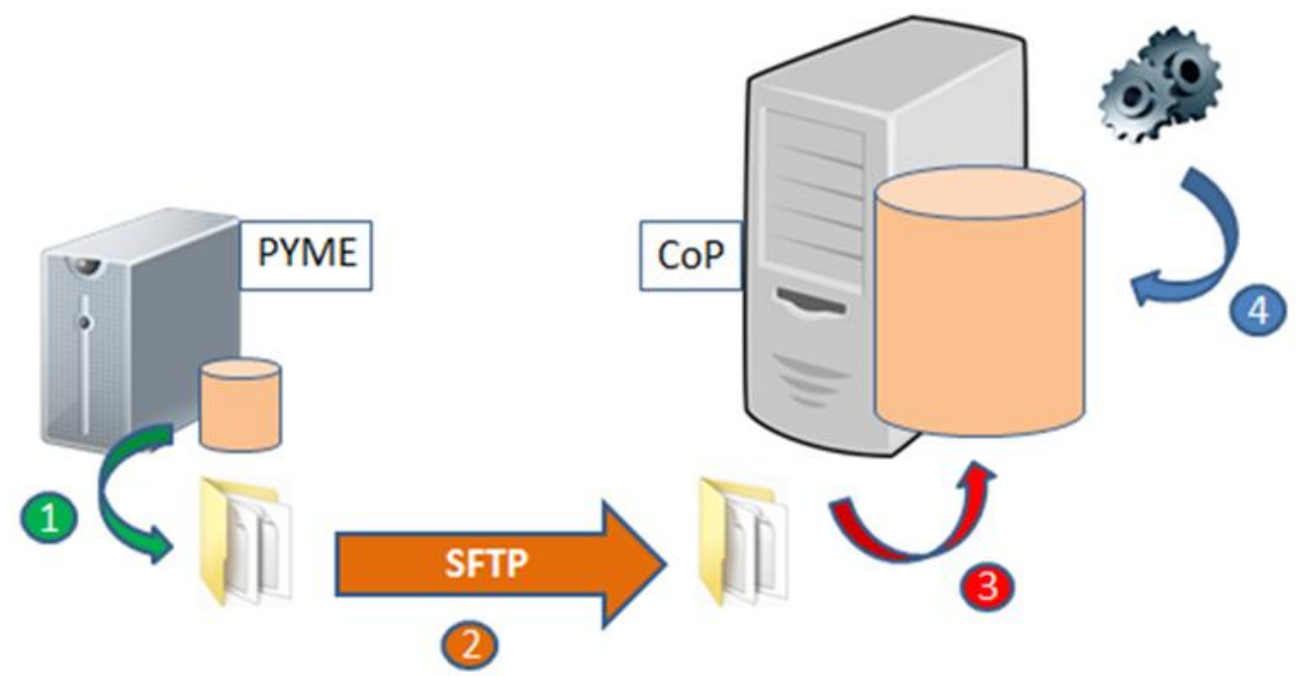

Figura 1. Metodología Sistemas de Monitoreo PYME - Comunidad de Práctica Figure 1. Methodology Monitoring Systems SME - Community of Practice Fuente: elaboración del autor. 


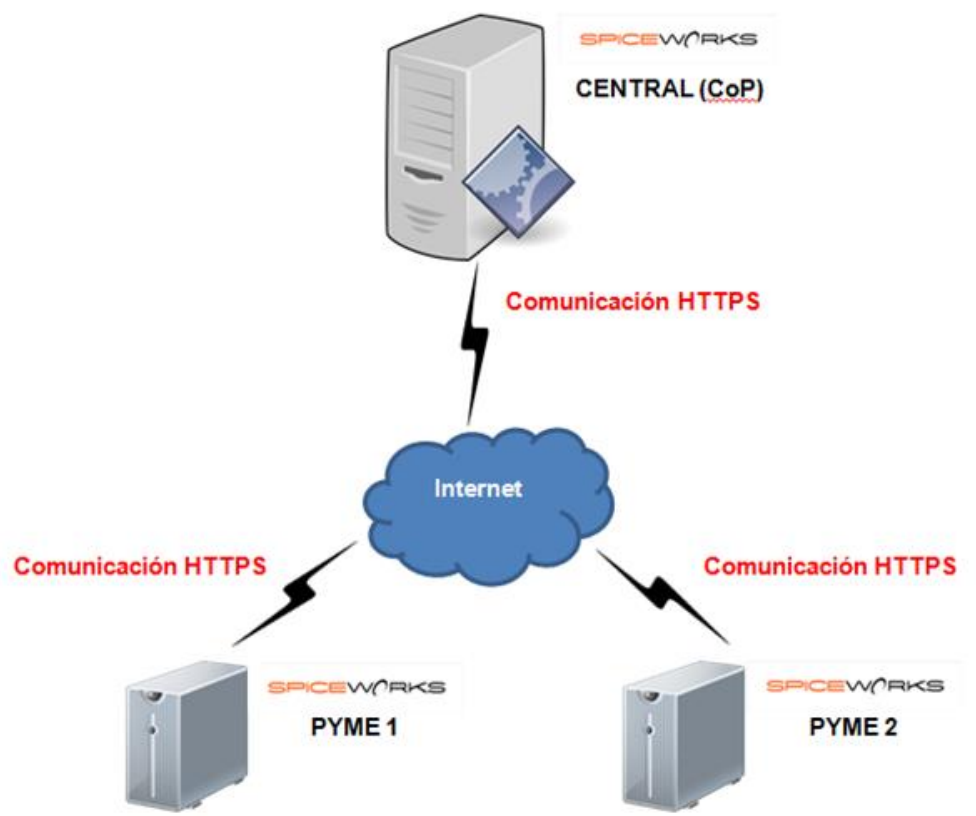

Figura 2. Arquitectura General

Figure 2. General architecture

Fuente: elaboración del autor.

La arquitectura propuesta se basó en una plataforma central, que es el nodo común entre todos los actores que pertenecen a la CoP, (ver Fig. 2). Cada instalación del software de monitoreo se conecta con la plataforma central con el fin de intercambiar la información que se quiere compartir, para el tema particular, información de inventario de hardware y software y la base de conocimiento construida a partir de la gestión de incidentes de IT.

Teniendo en cuenta los elementos generales en el trabajo desarrollado, se propuso un sistema de integración que ejecuta: el proceso de exportación de las tablas y registros necesarios para la integración, la transferencia de los archivos que contienen la información exportada desde el cliente (PYME) hacia el servidor (CoP), la ejecución de proceso de importación de los registros de la PYME a la base de datos de la CoP y la modificación de los datos de la PYME en la base de datos de la CoP para garantizar que son anónimos. Este proceso supone una integración asincrónica, unidireccional y con una periodicidad programable de acuerdo con las necesidades. Para garantizar que la información fluya con la inmediatez característica de las CoP, se planteó ejecutar el proceso de integración con una periodicidad de un (1) minuto.

\section{CONCLUSIONES}

Las PYME realizan procesos de administración de IT que normalmente responden a acciones correctivas, debido a que no cuentan con una cultura de prevención que los motive a usar herramientas de monitoreo y generación de alertas. Un sistema de monitoreo y administración de incidentes de IT le concede al administrador de IT tener un control más detallado sobre su área, ya que le permite generar indicadores de gestión y proponerse metas en períodos de tiempo. 
El administrador de IT evita participar en CoP debido al tiempo que puede tomarle dicha tarea y percibe poco valor de retorno debido a la ausencia de cultura de gestión del conocimiento. Este aspecto logra superarse gracias a un sistema automático de integración con una CoP a partir de su propio sistema de monitoreo y gestión de incidentes de IT.

La confidencialidad de la información es la intranquilidad más sobresaliente a la hora de mencionar la posibilidad de conformar un proceso de integración con una CoP. Sin embargo, presentando los argumentos técnicos que soportan la seguridad de la información y el carácter anónimo de la misma, se mitiga ampliamente este riesgo.

\section{REFERENCIAS}

Barragán, D. (2015). Las Comunidades de Práctica (CP): hacia una reconfiguración hermenéutica. Franciscanum, Ivii, 163, 155-176.

Cox, A. (2005). What are communities of practice? A comparative review of four seminal works. Journal of Information Science, 31(6), 527-540. doi:10.1177/0165551505057016.

De León, M.; Villa, J. L.; Vázquez, S. \& Salcedo, J. A. R. (2014). Description of information technologies adoption in small enterprises using the lazy-user model: A study case. RISTI - Revista Ibérica de Sistemas e Tecnologías de la Información, 1(E1), 91-104.

Driff, L. N. \& Guessoum, A. (2013). Social validation of learning objects in online communities of practice using semantic and machine learning technique.
Droschl, G. (2004). Communities of Practice: An Integrated Technology Perspective. Journal of Universal Computer Science, 10(3), 284-293.

Frezzo, D.; Cinque, G. \& Cinque, M. (2008). Use of Web 2.0 Technologies and Interaction Design to Enhance a Networking Instructor Community of Practice. Cisco Systems Inc.

Ghosh, B. \& Scott, J. E. (2009). Using a community of practice to enhance data quality in a distributed healthcare information system. ACIS 2009 Proceedings - 20th Australasian Conference on Information Systems.

Oficina de Comercio del Gobierno Británico (2011). ITIL - Gestión de Servicios de TI. Gobierno Británico 2011. Disponible en http://itil.osiatis.es.

Olszak, C. M. \& Ziemba, E. (2008). Communities of practice in knowledge management and organizational learning. Harnessing Knowledge Management to Build Communities - Proceedings of the 11th Annual Australian Conference on Knowledge Management and Intelligent Decision Support, ACKMIDS 08.

Preece, J.; Maloney-Krichmar, D. and Abras, C. (2003). History of Emergence of Online Communities. In B. Wellman (Ed.). Encyclopedia of Community. Berkshire Publishing Group, Sage.

Project Management Institute (PMI) (2008). Project Management Body of Knowledge (PMBOK). 4a Edición, 267, Project Management Institute, Inc, Pennsylvania, USA. 
Vásquez, S. (2011). Comunidades de Práctica. Europe Business School. Educar, 47, 5168. 